\title{
Sections and Shadows of Four-Dimensional Objects
}

\author{
Michal Zamboj ${ }^{1}$ (D)
}

\begin{abstract}
Methods of constructions of sections and shadows of four-dimensional objects are explained. We visualize images of an object and its section or shadow at the same time in a double orthogonal projection on two perpendicular three-dimensional spaces. Our techniques are similar to the methods used in Monge's projection of the three-dimensional space. Constructions are created in the interactive 3D modeling software GeoGebra 5, in which the user can freely move some input elements. We focus on a synthetic approach to description of the four-dimensional space with the use of descriptive geometry tools.
\end{abstract}

Keywords Four-dimensional visualization - Multidimensional descriptive geometry · Monge's projection · Four-dimensional polytope section · Fourdimensional shadow

\section{Introduction}

We introduce visual methods of examination of the four-dimensional space. Our goal is to develop instructive synthetic techniques with the use of descriptive geometry and interactive graphics to improve our spatial perception of fourdimensional objects. We follow the terminology and notation of Zamboj (2018), where the introduction into a synthetic method of projection of four-dimensional objects onto two perpendicular 3-spaces and further bibliography on the

Electronic supplementary material The online version of this article (doi:https://doi.org/10.1007/ s00004-018-0384-x) contains supplementary material, which is available to authorized users.

Michal Zamboj

zamboj@karlin.mff.cuni.cz

1 Faculty of Mathematics and Physics, Charles University, Sokolovská 83, 18675 Prague 8,

Czech Republic 
development of their visualization is given. Even though we explain all the ideas in the text, descriptive geometry constructions are led in technical language, which is hard to be followed without understanding of processes and proper definitions in the given reference. Specifically, we suggest the reader to go through the text with constructions and watch their corresponding interactive models in the supplementary material simultaneously.

To comprehend spatial properties of a three-dimensional solid drawn on a twodimensional paper, different strategies may be chosen. Various projections give us some basic information about the solid. However, if we also draw projections of intersections with planes or the shadow cast by the solid, we have much better understanding of its spatiality. In four dimensions we proceed analogically and construct visualizations of solids, their sections and shadows, up to one small difference-we cannot really imagine them as a whole.

To remind the method of double orthogonal projection, we start with a construction of a regular pentachoron. The nearest synthetic methods of visualization of the 4-space with using descriptive geometry are to be found in Lindgren and Slaby (1968) and Şerbănoiu and Şerbănoiu (2017). In both, an object is projected onto two-dimensional planes, and therefore we reconstruct the original image from three (by Lindgren and Slaby) or six (by Şerbănoius) planar images. It seems to be unbearable to carry three or more such images of complex objects, such as polychora, ${ }^{1}$ in mind and perform operations with them. Orthogonal projections are also used in Miyazaki (1988), where a rolling four-dimensional die is visualized. In contrast to parallel projection, in Séquin (2002) physical three-dimensional images of regular polychora in perspective projection are constructed. The properties of polychora are derived as natural analogies to the three-dimensional polyhedra. A famous treatise on regular polytopes as inductive dimensional analogies is in Coxeter (1973), Chapter VII. Nowadays, various models of polychora, in both parallel and central projections, may also be found all over the Internet. A very good example with an extensive encyclopedia is Eusebeîa (2018). Interesting method of distorted visualization of four-dimensional objects on an example of tesseract is proposed in Casas (1984). Casas developed a polar perspective-method of visualization of the four-space created by continuous compositions of perspective projections onto spheres, which are later flattened. Worth mentioning is also the investigation of polychora after decomposition into their three-dimensional net. Popular depiction of such net is Dalí's Crucifixion (Corpus Hypercubus) [(see Kemp (1998)].

Abbott in his Flatland Abbott (2006) describes an analogy when a 3-sphere appears as its circle sections in the flat two-dimensional world. Several approaches of visualizing cuts of four-dimensional objects by 3-spaces have been developed in computer graphics. A regular pentachoron is also the subject of investigation in Kageyama (2016). The pentachoron is given by analytic coordinates of its vertices, while multiple slices with parallel hyperplanes are computed and visualized in perspective projection, and then, they are placed on a parabolic curve. A similar process is applied to rotated positions of the polychoron for even better

\footnotetext{
${ }^{1}$ A polychoron is a four-dimensional polytope, generalization of a polyhedra.
} 
performance. The author also gives extensive literature on methods of investigation of four-dimensional objects in computer vision. A visualization of series of threedimensional slices of time-varying data, considered to be a four-dimensional data field, is shown in Woodring et al. (2003). All these computer graphics methods use analytic representations and computations. In this paper we proceed synthetically. Since we can properly visualize an object and its cutting hyperplane in the double orthogonal projection, we construct images of both-the object and its section.

There is no better motivation to investigate shadows of "the upper world" then Plato's Allegory of the Cave: "He will require to get accustomed to the sight of the upper world. And first he will see the shadows best, next the reflection of men and other objects in the water, and then the objects themselves; next he will gaze upon the light of the moon and the stars; and he will see the sky and the stars by night, better then the sun, or the light of the sun, by day?" Plato (1897) p. 320. Numerical descriptions and projections of shadows of four-dimensional regular polytopes in special positions are described in Chilton (1971, 1980). An exquisite interpretation of models of three-dimensional shadows and projections of a four-dimensional hypercube is in Segerman (2016), Chapter 3. Hanson and Heng (1991) conclude their work on four-dimensional lighting with a provocative question: "Can humans acquire facility with four dimensions with enough practice?" Although we cannot answer the question (we simply hope so), we add another training method. We derive visualizing techniques of constructions of shadows in parallel and central lightings as the analogy to classical methods of the three-dimensional descriptive geometry.

\section{A Regular Pentachoron}

A regular pentachoron is the four-dimensional generalization of a regular tetrahedron. It is a simplex with five vertices, which consists of five regular tetrahedral cells. In three dimensions, a regular tetrahedron is easily created, when we join the vertices of an equilateral triangle with the fourth vertex, which is the endpoint of the altitude perpendicular to the triangle in its centre. Imagine, for illustration, that we want to introduce the tetrahedron to a geometrically educated two-dimensional creature. It will not be able to visualize the tetrahedron in mind, but still, we can draw its parallel projections onto a plane. If we draw the top and front views and merge them into a one picture, we obtain the descriptive geometry method of visualization called Monge's projection. Rather then trying to persuade the creature to imagine the missing dimension, we show projections of the tetrahedron, which describe it uniquely. After all, together with the creature, we can use basic descriptive geometry techniques (such as a rotation around a line) to investigate incidence and metric properties in the 3-space on the two-dimensional drawing paper. The construction of the tetrahedron would start with the base equilateral triangle. Both horizontal and vertical images of the triangle would appear distorted in an affinity implied by the parallel projection. To see the triangle in its true shape, we would rotate it into the vertical projecting plane around the line of its intersection with the plane of the triangle. For the creature, this would seem like the 
axial affinity in the drawing plane, while the rotation around a line is impossible. Apparently, the planar creature would understand the concept of planar triangle and also the relation of perpendicularity between two lines. In Monge's projection, to construct a line perpendicular to a plane, we only need perpendicularity of lines in the drawing plane. For measurements we also use rotation and hence, we can continue to construct the altitude and the fourth vertex. After connecting all the vertices of the triangle with the fourth vertex, both projections would seem like four connected triangles (faces) for the creature not understanding the "insideness". However, it can measure and further investigate the polyhedron. Analogically, we project the two-dimensional creature into ourselves, while we generalize the construction for the regular pentachoron. In the double orthogonal projection, the four-dimensional pentachoron is projected onto two mutually perpendicular 3 -spaces. Instead of the drawing plane, we merge these spaces into a modeling 3-space. At first, we select a 3-space of the tetrahedral base. The orthogonal projections of this tetrahedron are affinely distorted, and therefore, we find the tetrahedron in its true shape after a rotation of its 3-space around the plane of intersection with one of the spaces of projection onto the modeling space. This rotation around the plane in the 4-space appears as affinity in the modeling 3-space. After construction of the rotated regular tetrahedral base in its true shape, we find its original image with use of the spatial axial affinity. Then, we construct the altitude of the pentachoron between the fifth vertex and the constructed base tetrahedron. The length of the altitude may be computed analytically or by inductive analogy from the regular tetrahedron. For construction of a line perpendicular to a 3-space in the double orthogonal projection, we only need a perpendicularity of lines and planes. The length of the altitude appears distorted, but again, we can measure the true length of the rotated image. After the construction of the fifth vertex, we connect all the vertices of the pentachoron, and the resulting images appear as compositions of five distorted tetrahedra.

A step-by-step construction on the interactive model in GeoGebra 5 is in Online Resource 1 (Fig. 1). Let us have a regular pentachoron $A B C D E$ with the cell $A B C D$ in the 3 -space $\Sigma$. In our example, the 3 -space $\Sigma$ is given by its traces $\xi^{\Sigma}$ and $\omega^{\Sigma}$. The $\Omega$-image $A_{4}$ of the point $A$ is given. The face $A B C$ is parallel to the $\Omega$-trace with the edge $A B$ parallel to the reference plane $\pi(x, y)$.

We start with the construction of the tetrahedron $A B C D$. For the construction of the $\Xi$-images of the points $A$ and $B$ we use the $\Omega$-plane of the 3 -space $\Sigma$ (not shown in the figure). The tetrahedron appears in its true shape $A_{0} B_{0} C_{0} D_{0}$ in the image $\Sigma_{0}$ of the space $\Sigma$ after its rotation around the $\Omega$-trace $\omega^{\Sigma}$ onto the modeling space [see Zamboj (2018), pp. 11-12, Figure 10]. With the edge $A_{0} B_{0}$ we can finish the construction of the regular tetrahedron $A_{0} B_{0} C_{0} D_{0}$. In the affinity between $\Sigma_{4}$ and $\Sigma_{0}$ we construct the $\Omega$-images $C_{4}$ and $D_{4}$. On the lines of recall and the $\Omega$-planes of the 3 -space $\Sigma$ we find the $\Xi$-images $C_{3}$ and $D_{3}$.

Further on, we need to find the projections of the point $E$. Parallel projections preserve centres of segments, and therefore also the centroid of a triangle or tetrahedron. Since the pentachoron $A B C D E$ is regular, the vertex $E$ lies on the perpendicular line to the 3 -space $\Sigma$ through the centroid $T$ of the tetrahedron $A B C D$, 


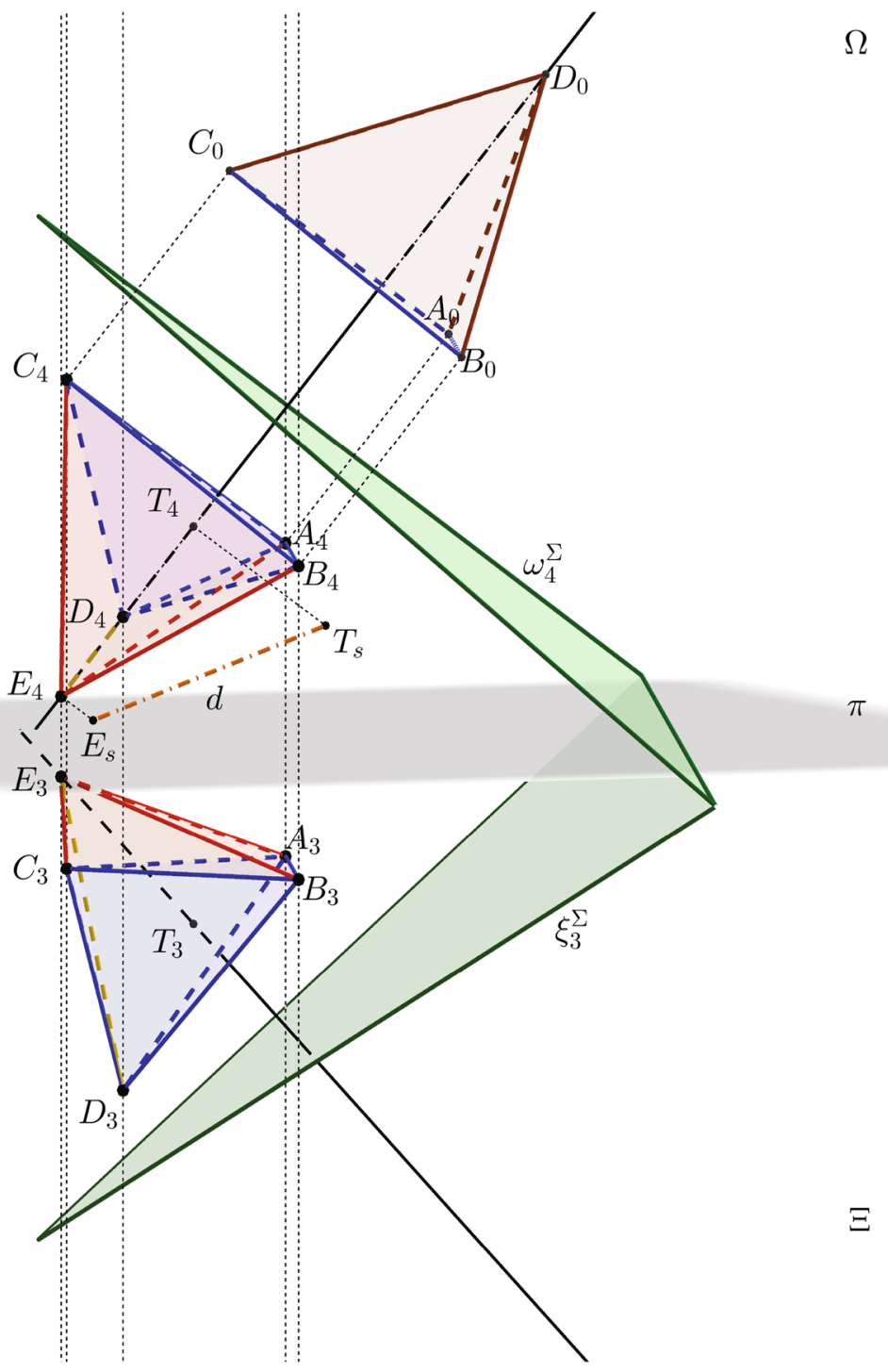

Fig. 1 A construction of a regular pentachoron

and the true length of the segment $T E$ can be easily computed as $d=|T E|=\frac{|A B|}{2} \sqrt{\frac{5}{2}}$. Therefore, the points $E_{3}$ and $E_{4}$ lie on the lines through $T_{3}$ perpendicular to $\xi_{3}^{\Sigma}$ and through $T_{4}$ perpendicular to $\omega_{4}^{\Sigma}$, respectively. ${ }^{2}$ The segment $T E$ appears in its true shape $T_{s} E_{s}$, with the length $\left|T_{s} E_{s}\right|=d$, after some rotation of the $\Omega$-projecting plane of $T E$ around the $\Omega$-image $T_{4} E_{4}$ onto the modeling space [see Zamboj (2018), pp.

\footnotetext{
${ }^{2}$ The centroids $T_{3}$ of the tetrahedron $A_{3} B_{3} C_{3} D_{3}$ and $T_{4}$ of the tetrahedron $A_{4} B_{4} C_{4} D_{4}$ are constructed in the GeoGebra model automatically, even though their synthetic construction is simple.
} 
10-11, Figure 8]. Hence, the point $E_{4}$ is found as the original point of the point $E_{s}$. The $\Xi$-image $E_{3}$ lies on the line of recall of the point $E$.

Connecting the tetrahedra $A B C D, A B C E, A B D E, A C D E$ and $B C D E$ in their $\Xi$ and $\Omega$-images, the pentachoron $A B C D E$ is constructed.

\section{A Construction of a Section Cut of a Cubic Pyramid}

In the following section we cut a four-dimensional regular pyramid with a cubical base with a 3-space. The chosen polychoron may be considered as a simple analogy to a right square pyramid. In Monge's projection onto a two dimensional drawing plane, if the square pyramid stands with its base on the horizontal ground, the top view of the base square appears in its true shape and the image of the vertex is in its centre. The front view of the square appears as a segment, and the distance between the segment and the vertex is the true length of the altitude. This placement is convenient for cutting the square pyramid with a plane perpendicular to the plane of the vertical plane of projection. The section in the front view is only a segment, and its image in the horizontal plane is a quadrilateral, which is a collinear image of the base square. In the 4-space we place the regular cubic pyramid analogically with respect to the pair of perpendicular 3-spaces. The pyramid stands with the base cube in one of the spaces and its altitude is perpendicular to the cube. Therefore, in the first view, the base cube appears in its true shape, and the image of the vertex is its centre. In the second perpendicular view, the image of the cube merges into a square, and the image of the whole cubic pyramid seems as a three-dimensional square pyramid. The cubic pyramid is in a good position to be cut with a 3-space. We choose the cutting space similarly as in Monge's projection above. Hence, one image of the section is a quadrilateral (instead of the segment), and the second is a hexahedron (instead of quadrilateral). The hexahedral image of the section is again a collinear image of the base cube.

In the interactive model (Online Resource 2), we can rotate the cutting 3-space around its intersecting line with the reference plane by manipulating the $\Omega$-trace of the cutting 3-space.

Let us construct a cubic pyramid $A B C D E F G H V$ with the base cube $A B C D E F G H$ in the 3-space $\Xi(x, y, z)$ and with the face $A B C D$ in the reference plane $\pi(x, y)$ (Fig. 2). Let the vertex $V$ lie on the perpendicular line to the cube through its center $S$ ( $S V$ is the altitude). We will describe the construction of the section cut, hexahedron $A^{\prime} B^{\prime} C^{\prime} D^{\prime} E^{\prime} F^{\prime} G^{\prime} H^{\prime}$, of the cubic pyramid by a 3 -space $\Sigma$ perpendicular to $\Omega(x, y, w)$ given by its traces $\xi^{\Sigma}, \omega^{\Sigma}$.

The given altitude $S V$ is perpendicular to the 3 -space $\Xi(x, y, z)$, hence the $\Xi$ images $S_{3}, V_{3}$ merge into one point in $\Xi(x, y, z)$. The $\Xi$-image $A_{3} B_{3} C_{3} D_{3} E_{3} F_{3} G_{3} H_{3}$ of the base cube appears in its true shape. The $\Xi$-image of the cubic pyramid appears as the union of six pyramids $A_{3} B_{3} C_{3} D_{3} V_{3}, A_{3} B_{3} F_{3} E_{3} V_{3}, \quad B_{3} C_{3} G_{3} F_{3} V_{3}$, $C_{3} D_{3} H_{3} G_{3} V_{3}, D_{3} A_{3} E_{3} H_{3} V_{3}, E_{3} F_{3} G_{3} H_{3} V_{3}$. On the other hand, the $\Omega$-image of the cube $A B C D E F G H$ appears as the square $A_{4} B_{4} C_{4} D_{4} \equiv E_{4} F_{4} G_{4} H_{4}$ in $\Omega(x, y, w)$. Thus, the $\Omega$-image of the cubic pyramid is the pyramid $A_{4} B_{4} C_{4} D_{4} V_{4}$. 


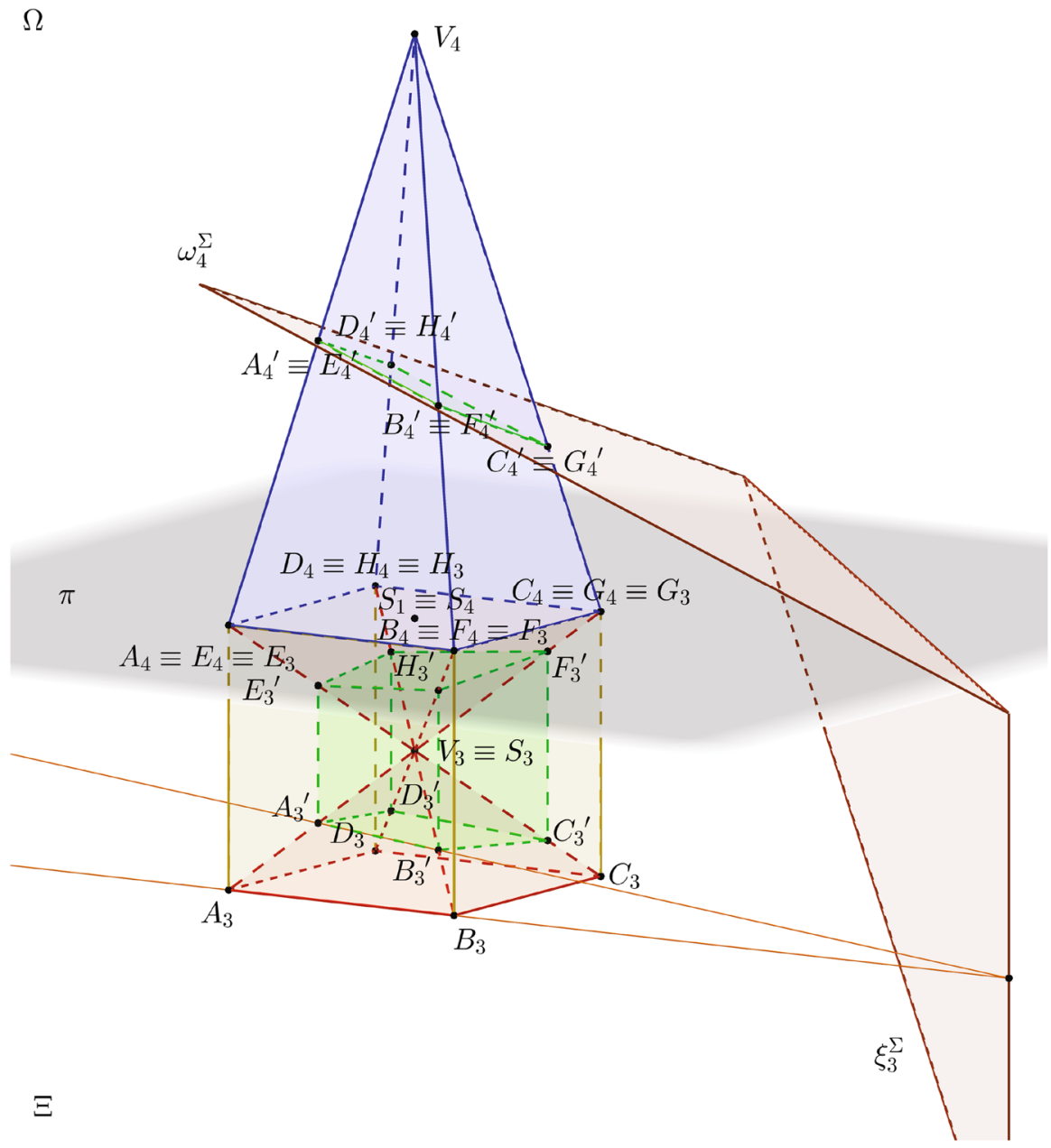

Fig. 2 A construction of a section cut of a cubic pyramid with a 3-space

The $\Omega$-image of the section is the quadrilateral $A_{4}^{\prime} B_{4}^{\prime} C_{4}^{\prime} D_{4}^{\prime} \equiv E_{4}^{\prime} F_{4}^{\prime} G_{4}^{\prime} H_{4}^{\prime}$ lying in the $\Omega$-trace $\omega_{4}^{\Sigma}$ of $\Sigma$, where the points $A_{4}^{\prime}, B_{4}^{\prime}, C_{4}^{\prime}, D_{4}^{\prime}, E_{4}^{\prime}, F_{4}^{\prime}, G_{4}^{\prime}, H_{4}^{\prime}$ are the intersections of the plane $\omega_{4}^{\Sigma}$ and the edges $A_{4} V_{4}, B_{4} V_{4}, C_{4} V_{4}, D_{4} V_{4}, E_{4} V_{4}, F_{4} V_{4}, G_{4} V_{4}, H_{4} V_{4}$, respectively. To construct the $\Xi$-image of the section we can conveniently use lines of recall or the perspective collineation between two 3-spaces: $\Sigma$ - the cutting 3-space and $\Xi(x, y, z)$ - the 3 -space of the base cube. The center of the collineation is the point $V$ and the axial plane of the collineation is the $\Xi$-trace $\xi^{\Sigma}$ of the cutting 3 -space $\Sigma$. The construction of the image $B_{3}^{\prime}$ of the point $B_{3}$ in the collineation, with the use of the fixed point of the line $A_{3} B_{3}$, is visualized in the figure. The same process is applied to find the remaining vertices of the hexahedron $A^{\prime} B^{\prime} C^{\prime} D^{\prime} E^{\prime} F^{\prime} G^{\prime} H^{\prime}$.

Section cuts, which are obtained by rotating the cutting 3 -space around its line of intersection with the reference plane, are shown in Fig. 3. 


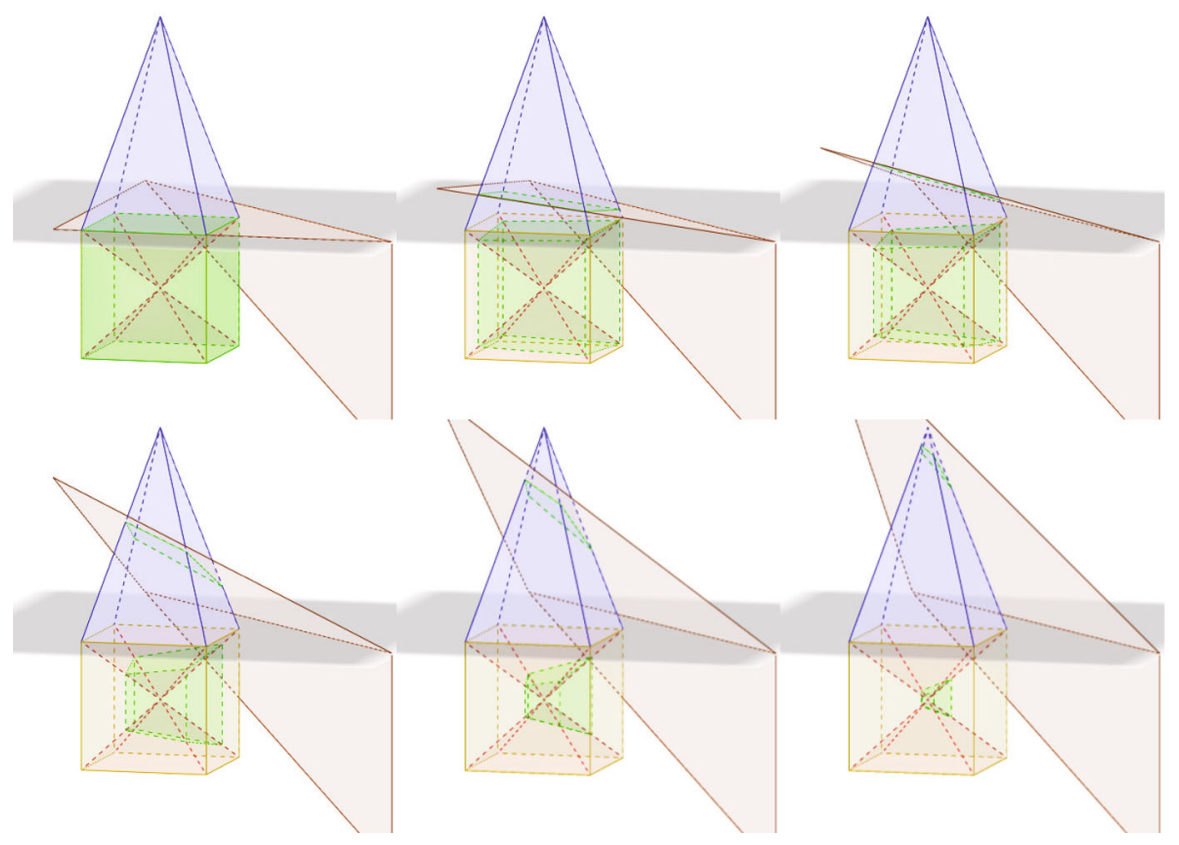

Fig. 3 Development of section cuts of a cubic pyramid with a rotating 3-space

\section{Constructing Shadows}

To examine objects in the 4-space, we can construct their three-dimensional shadows. When we return to the idea of the two dimensional creature, we can think about its perception of a shadow. It would probably understand that a twodimensional object, illuminated from either finite or infinite point source, casts a shadow on a line. The shadow is a mere projection of the object onto the line, and we construct it as the set of intersection points of the light rays with this line. Similarly, our concept of a shadow is a two-dimensional projection, planar image, of a three-dimensional object. Hence, for example, the shadow that the square pyramid casts on the plane of the creature is in its visual possibilities (recall Plato). In Monge's projection we merge the vertical and the horizontal plane into one drawing plane, and we construct shadows cast on both of them. If the shadow of the object breaks in their edge-their line of intersection, it appears broken in the drawing plane, too. The geometric constructions are realized in the drawing plane, and therefore, the two-dimensional creature is able to apply them. At last, the fourdimensional story is similar. At this point, we can visualize a four-dimensional polychora, light rays and also their intersections with the given perpendicular 3 -spaces, which form its three-dimensional shadows. Instead of the edge line, the shadow may break in the edge plane, which is the plane of intersection of these spaces. Certainly, the following methods are another analogy of visualizing the 3 -space in a plane and constructing shadows of three-dimensional objects. For methods of constructing shades and shadows in the three-dimensional descriptive 


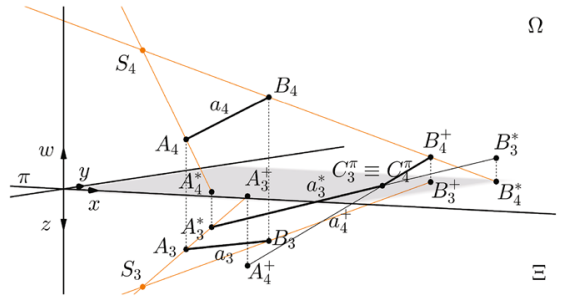

(a)

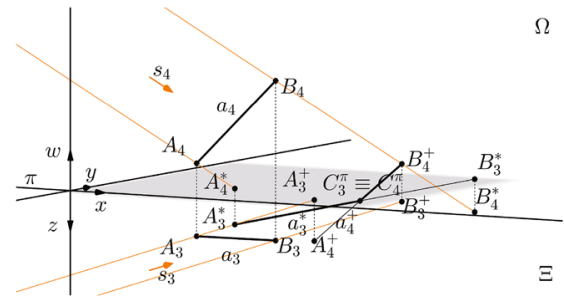

(b)

Fig. 4 a A shadow of a segment $A B$ in a central lighting. b A shadow of a segment $A B$ in a parallel lighting

geometry on various examples see classical textbooks on descriptive geometry such as Warren (1867). We introduce central and parallel lighting techniques on illumination of a segment, and afterwards, we construct the shadow of the cubic pyramid described in the previous section.

\section{Parallel and Central Lighting}

The shadow of a segment in both central and parallel lighting is constructed below as the set of points of intersection of light rays with the given pair of perpendicular 3 -spaces of projection. Shadows cast on both 3-spaces are again segments, which intersect in the edge plane in our examples. To construct the segments, we only need to find the shadows of their end points. The final shadow of the segment appears as a broken line in the modeling space.

Let us have a segment $A B$ and a point source of light $S$ in the 4-space (Fig. 4a). The segment $A B$ casts shadows $A^{*} B^{*}$ and $A^{+} B^{+}$on the 3 -spaces $\Xi(x, y, z)$ and $\Omega(x, y, w)$, respectively.

The shadow $A^{*} B^{*}$ is the intersection of light rays from the source $S$ through the points of the segment $A B$ with the 3 -space $\Xi(x, y, z)$. Therefore, the $\Omega$-image $A_{4}^{*} B_{4}^{*}$ lies in the reference plane $\pi(x, y)$. To find the $\Xi$ and $\Omega$-images of the point $A^{*}$ we construct the ray $S_{4} A_{4}$, which intersects the reference plane $\pi(x, y)$ in $A_{4}^{*}$. The point $A_{3}^{*}$ lies on the ray $S_{3} A_{3}$ and on the line of recall through the point $A_{4}^{*}$. The $\Xi$ and $\Omega$ images of the point $B^{*}$ are constructed in the same fashion.

The $\Xi$-image of the shadow $A^{+} B^{+}$of the segment $A B$ cast on the 3-space $\Omega(x, y, w)$ lies in the reference plane $\pi(x, y)$. Thus, the point $A_{3}^{+}$is the intersection of the ray $S_{3} A_{3}$ and the reference plane $\pi(x, y)$. Its $\Omega$-image $A_{4}^{+}$lies on the line of recall and on the ray $S_{4} A_{4}$. With a similar construction we finish the images of the point $B^{+}$and the shadow $A^{+} B^{+}$.

The shadow may break in the edge plane-the reference plane $\pi(x, y)$. If this is the case, we show only the parts of the shadow in the visible half-3-spaces ${ }^{3}$ of $\Xi(x, y, z)$ and $\Omega(x, y, w)$. Our shadows $A^{*} B^{*}$ and $A^{+} B^{+}$intersect in the point $C^{\pi}$, in which the shadow of the segment $A B$ is broken.

\footnotetext{
${ }^{3}$ The half-3-spaces of $\Xi(x, y, z)$ with a nonnegative $z$ coordinate and $\Omega(x, y, w)$ with a nonnegative $w$ coordinate are visible.
} 
If the source of light is given by a direction $s$ (Fig. 4b), the lighting is parallel. Instead of the rays through the point $S$ we need rays parallel to the direction $s$, otherwise the construction is similar to the central lighting.

\section{A Construction of a Shadow of a Cubic Pyramid}

Previously, we placed the right cubic pyramid to a convenient position for the construction of its sections. We use the same setting for the construction of its shadow. Now, we again skip back to the lower dimension and imagine that the twodimensional creature is in the same plane as the square base of a right square pyramid. Hence, the whole pyramid appears simply as the square. Furthermore, let there be a point light source, which is not in the creature's plane. When the square pyramid is illuminated from the light source, it casts a shadow on the base plane. The shadow of the base square is obviously the base square itself. To construct the rest of the shadow, the creature needs only to find the shadow of the vertex of the pyramid. After connecting the shadow of the vertex and the base square, the construction is finished. This is the main idea of the first step of our fourdimensional construction. The base cube of the cubic pyramid lies in one of the 3 -spaces of projection and it overlaps its shadow cast on this 3-space. We only find the shadow of the vertex and join it with the base cube. In our example in the double orthogonal projection, the shadow breaks in the reference plane, thus we construct the shadow of the vertex also in the second perpendicular 3-space, and connect it with the break points of the sides of the cubic pyramid. Finally, the shadow cast on the two 3-spaces looks like a broken polyhedron.

In this last construction (Fig. 5, Online Resource 3), we use the same model of the cubic pyramid $A B C D E F G H V$ as in "A Construction of a Section Cut of a Cubic Pyramid". Let us have a point $S$, the point light source, given by its $\Xi$ and $\Omega$-images. The cubic pyramid, illuminated from the source $S$, casts shadows on the 3 -spaces $\Xi(x, y, z)$ and $\Omega(x, y, w)$. Firstly, we identify the shade of the cubic pyramid. The surface of shadow is the shadow of its surface of shade. ${ }^{4}$ From the $\Xi$-image we see that the squares $A B C D, A B F E, D A E H$ are illuminated. From the $\Omega$-image we see the illuminated faces $A B V, A E V, B F V, E F V, D A V, E H V, H D V$. The rest of the cubic pyramid, the union of cells $B C G F V, C D H G V, E F G H V$, is the shade. The boundary of the shade-faces $B C G F, C D H G, E F G H, B C V, C D V, D H V, H E$ $V, E F V, F B V$ forms the surface of shade. For the clarity of visualization, only those parts of the shadow, which do not intersect the projections of the body, are constructed.

The shadow of the squares $B C G F, C D H G, E F G H$ on the 3 -space $\Xi(x, y, z)$ remains their $\Xi$-projections $B_{3} C_{3} G_{3} F_{3} \equiv B_{3}^{*} C_{3}^{*} G_{3}^{*} F_{3}^{*}, C_{3} D_{3} H_{3} G_{3} \equiv C_{3}^{*} D_{3}^{*} H_{3}^{*} G_{3}^{*}$, $E_{3} F_{3} G_{3} H_{3} \equiv E_{3}^{*} F_{3}^{*} G_{3}^{*} H_{3}^{*}$, since they lie in the 3 -space $\Xi(x, y, z)$. To construct the shadow of the vertex $V$, we find the point of intersection $V^{*}$ of the light ray $S V$ with the 3-space $\Xi(x, y, z)$ according to Fig. 4a. Further on, we construct the shadow of the edge $B V$ by joining $B_{3}^{*}$ and $V_{3}^{*}$. In our setting, the shadow of the edge $B_{3} V_{3}$

\footnotetext{
${ }^{4}$ This is a generalization of the famous descriptive geometry theorem: The curve of shadow is the shadow of its curve of shade.
} 


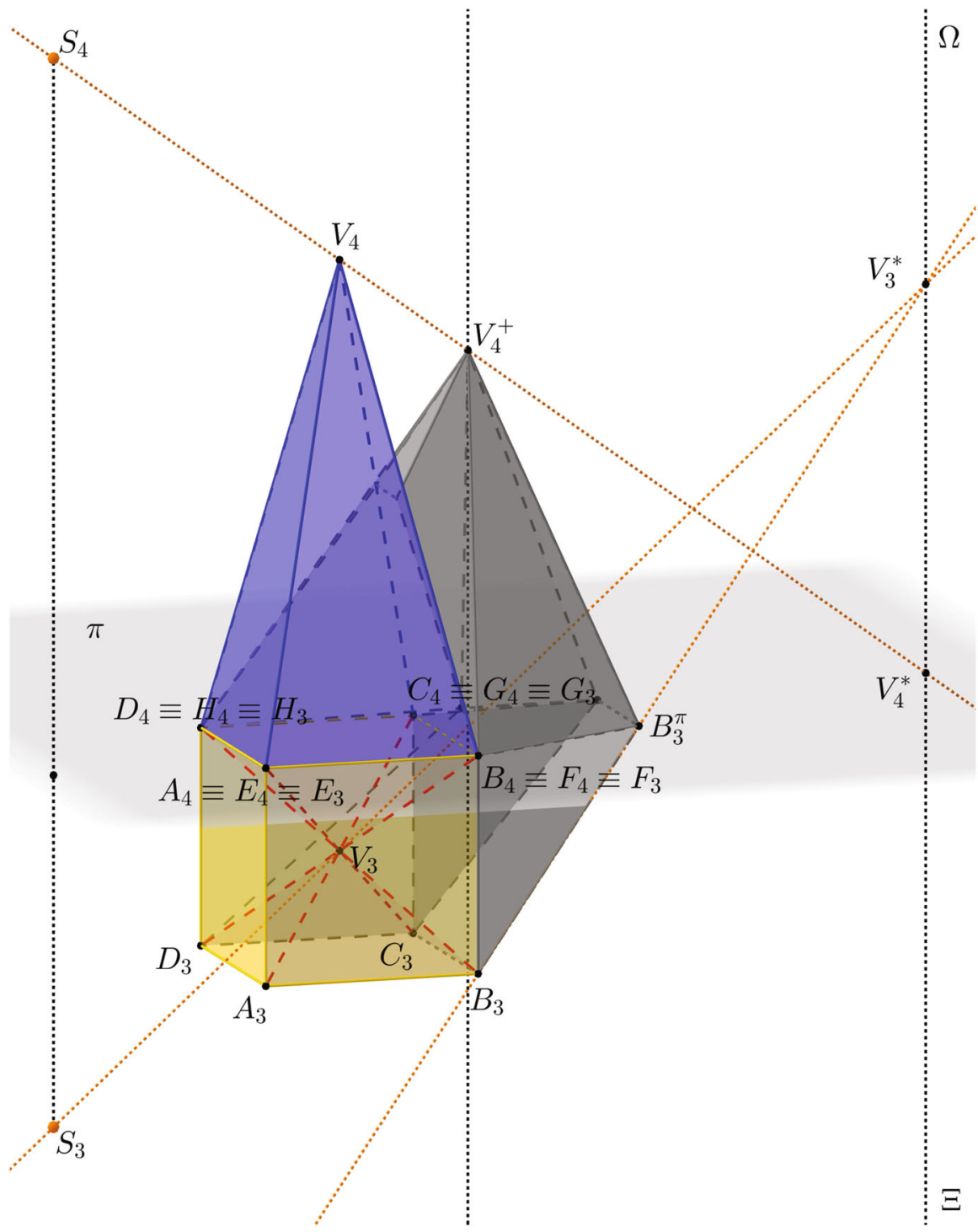

Fig. 5 A construction of the shadow of a cubic pyramid on the 3 -spaces $\Xi(x, y, z)$ and $\Omega(x, y, w)$. The full construction of the points $V$ and $B$ is shown

intersects the reference plane $\pi$ in the point $B_{3}^{\pi}$, where the shadow breaks and continues in the 3 -space $\Omega(x, y, w)$. The rest of the shadow in the 3 -space $\Xi(x, y, z)$ can be constructed analogically.

The shadow cast on the 3-space $\Omega(x, y, w)$ can be constructed in the same manner, but we can also conveniently use the breaking points of the shadow in the reference plane $\pi(x, y)$. Therefore, the last missing point is the shadow $V^{+}$of the vertex $V$, which is the intersection of the 3 -space $\Omega(x, y, w)$ and the light ray $S V$. 
Finally, we only need to join all the points of the shadow in the reference plane $\pi(x, y)$ such as $B_{3}^{\pi}$ with $V_{4}^{+}$. The images of the final shadow are three-dimensional solids in the modeling space.

\section{Conclusion}

Studies on visualization of objects in the four-dimensional space usually depend on analytic representations. We have described a synthetic approach with a possibility of interactive manipulation. Common methods, such as projecting sections or shadows were introduced in a double orthogonal projection onto two perpendicular three-dimensional spaces. Furthermore, we have shown images of a section or shadow in the same model (or figure) with the projections of an original object. Even though, we emphasize the synthetic point of view on the four-dimensional geometric space in this article, we are not opposed to analytic methods of visualization and examination. While analytic representations are valuable for verifications, the synthetic approach leads to intuition and further creations. There is one more question, which often arises in discussions about visualizations of the four-dimensional space. What is their usefulness? Firstly, didactical essence of meditations about the fourth dimension is in abstraction of the ideal geometric space. We mostly use analogies of methods empirically known from the two and 3 -space. But, neither our spatial ability nor geometric space ends where $n$ equals 3 . Furthermore, if we can describe the four-dimensional space, we should use all the possible methods to examine it. When the doors to the room of the fourth dimension are open, why should we not walk inside and find all its mysteries. Last, but not least, is the aspect of applications. Last 150 years human science has tested many hypothesis based on multidimensionality. Four-dimensional visualizations are now used in physics, computer science and other mathematically related sciences. Knowledge and experience with the four-dimensional space is essential for searching for answers and asking further questions about the world we live in, and where is the limit of the architecture of four-dimensional environments of such applications?

Acknowledgements The work was supported by the grant SVV No. 260454. I would also like to thank the independent referees of the Nexus Network Journal for their useful suggestions and patient proof reading of the technical parts of this and previous paper.

\section{References}

Abbott, Edwin Abbott. 2006. Flatland, a Romance of Many Dimensions. Edited with an Introduction and Notes by Rosemary Jann, Oxford University Press.

Casas, Fernando R. 1984. Polar Perspective: A Graphical System for Creating Two-Dimensional Images Representing a World of Four Dimensions. Leonardo 17, 3: 188-194. https://doi.org/10.2307/ 1575189

Chilton, Bruce L. 1971. Shadows of Four-Dimensional Polytopes. Mathematics Magazine 44, 5: 269-273. https://doi.org/10.2307/2688637 
Chilton, Bruce L. 1980. Principal Shadows of the 12 Pentagonal Regular 4-Dimensional Objects (Polytopes). Leonardo 13, 4: 288-294. https://doi.org/10.2307/2688637

Coxeter, Harold Scott MacDonald. 1973. Regular Polytopes. Third edition, Dover Publications, Inc.

Eusebeîa, http://eusebeia.dyndns.org/4d/ (accessed 29 March 2018)

Hanson, Andrew J. and Pheng Ann Heng. 1991. Visualizing the Fourth Dimension Using Geometry and Light. In: Proceedings of the 2nd conference on Visualization '91: 321-328. https://doi.org/10.1109/ VISUAL.1991.175821

Kageyama, Akira. 2016. A Visualization Method of Four Dimensional Polytopes by Oval Display of Parallel Hyperplane Slices. Journal of Visualization 19, 3: 417-422. https://doi.org/10.1007/s12650015-0319-5

Kemp, Martin. 1998. Dali's dimensions. Nature 391: 27. https://doi.org/10.1038/34063

Lindgren, Carlos Ernesto S. and Steve M. Slaby. 1968. Four-Dimensional Descriptive Geometry. New York: McGraw-Hill.

Miyazaki, Koji. 1988. Visualization of a Die and Other Objects in Four-Dimensional Space. Leonardo 21, 1: 56-60. https://doi.org/10.2307/1578417

Plato. 1897. The Republic [Books I-X] In: Plato the teacher: Being selections from the Apology, Euthydemus, Protagoras, Symposium, Phadrus, Republic, and Phado of Plato, eds. W. L. Bryan and C. L. Bryan, 187-409, New York: Charles Scribner's Sons. https://doi.org/10.1037/14227-012

Segerman, Henry 2016. Visualizing Mathematics with 3D Printing. Johns Hopkins University Press.

Séquin, Carlo Heinrich. 2002. 3D Visualization Models of the Regular Polytopes in Four and Higher Dimensions. In: Proceedings of Bridges: Mathematical Connections in Art, Music, and Science: 37-48.

Şerbănoiu, Bogdan V., and Adrian A. Şerbănoiu. 2017. Multidimensional Descriptive Geometry. In: 16th edition National Technical-Scientific Conference, Modern Technologies for the 3rd Millennium, Romania: 257-266.

Warren, Samuel Edward. 1867. General problems of shades and shadows. New York: John Wiley and Sons.

Woodring, Jonathan L., Chaoli Wang and Han-Wei Shen. 2003. High dimensional direct rendering of time-varying volumetric data. In: IEEE Transactions on Ultrasonics, Ferroelectrics and Frequency Control, IEEE, Seattle, WA, USA. https://doi.org/10.1109/VISUAL.2003.1250402 http:// ieeexplore.ieee.org/document/1250402/ (accessed 08 December 2003)

Zamboj, Michal. 2018. Double Orthogonal Projection of Four-Dimensional Objects onto Two Perpendicular 3-spaces. In: Nexus Network Journal. https://doi.org/10.1007/s00004-017-0368-2 https://link.springer.com/article/10.1007/s00004-017-0368-2 (accessed 16 January 2018)

Michal Zamboj is a Ph.D. student and lecturer at the Charles University in Prague, currently on a study stay at the Technical University in Vienna. The main field of his research is projective, synthetic, descriptive geometry and graphics, and their connections. His second research interest lies in the mathematics of juggling, on which he wrote his bachelor and diploma theses. 